\title{
Influence of laterality on endometriosis severity in patients with unilateral endometrioma: a retrospective study
}

Wataru Isono ( $\square$ tetuken2010@gmail.com )

Teikyo University https://orcid.org/0000-0003-4589-328X

Michiko Honda

Teikyo University

Akira Tsuchiya

Teikyo University

Ako Saito

Teikyo University

Hiroko Tsuchiya

Teikyo University

Reiko Matsuyama

Teikyo University

Akihisa Fujimoto

Teikyo University

Osamu Nishii

Teikyo University

\section{Research article}

Keywords: Endometrioma, Laparoscopic Surgery, Multivariate Analysis, Retrospective Study, Severity of IIIness Index

Posted Date: February 7th, 2020

DOl: https://doi.org/10.21203/rs.2.22842/v1

License: (c) (i) This work is licensed under a Creative Commons Attribution 4.0 International License. Read Full License 


\section{Abstract}

Background: To evaluate the influence of the location of endometrioma (right vs. left ovary) on endometriosis severity, we compared the revised American Society for Reproductive Medicine (ASRM) scores determined by laparoscopic surgery.

Methods: The medical records of 151 patients, including 58 right-sided and 93 left-sided cases, who underwent initial laparoscopic surgery for unilateral ovarian endometrioma were reviewed retrospectively. We extracted the ASRM scores determined during surgery and some representative factors relating to endometirosis that are separate from tumour characteristics, such as the coexistence of uterine fibroid, adenomyoma, or other ovarian tumours, a history of assisted reproductive technology (ART), and tumour marker values. Multivariate analysis was performed to assess the impact of each factor. We classified the ASRM scores into 4 categories to compare the tendency towards endometrial lesion spread with a focus on the "sidedness" of the endometrioma. Opposite endometrial lesion (OEL), Douglas pouch endometrial lesion (DEL), same endometrial lesion (SEL) and peritoneal endometrial lesion (PEL) were defined.

Results: The ASRM scores of patients with right-sided endometrioma were significantly higher than those of patients with left-sided endometrioma ( $41.5 \pm 22.0$ points vs. $33.5 \pm 16.3$ points, $p<0.05)$. The higher ASRM scores of the patients with right-sided endometrioma may be caused by the frequent detection of endometrial lesions in the left-sided tube, left-sided ovary and Douglas pouch. Multivariate analysis revealed that the coexistence of uterine fibroid or adenomyoma also had significant positive impacts on increasing the ASRM score.

Conclusions: Higher ASRM scores are possible in patients with right-sided endometrioma. The laterality of endometriosis severity should be considered.

\section{Background}

Endometriosis is one of the most common gynaecologic diseases and is known to affect $2-10 \%$ of reproductive-age women [1,2]. While many patients are asymptomatic [3], endometriosis is frequently associated with infertility and pain symptoms, including chronic pelvic pain, dysmenorrhoea, dyspareunia, and dyschezia [4]. Previous studies have revealed a relationship between symptom severity and disease stage according to the revised American Society for Reproductive Medicine (ASRM) scoring system [5]. In most cases, the diagnosis of endometrioma is made by outpatient ultrasound examination [6], and laparoscopic surgeries are often performed to treat pelvic pain and subfertility [7]. During surgery, ovarian endometrioma, superficial implantation and deep infiltrating endometriosis are detected. Additionally, some tendencies in endometriosis distribution have been indicated [8]. Some studies have suggested that left-sided endometriomas are more common than right-sided endometriomas [9]. This tendency is believed to occur due to anatomical asymmetries in the pelvic organs, including the presence of the sigmoid colon [9]. However, the recognition of this laterality is insufficient. Therefore, we aimed not 
only to verify this laterality but also to evaluate the influence of the side of endometrioma on disease severity. Specifically, the ASRM scores determined during laparoscopic surgery were compared.

\section{Methods}

\section{Data collection}

This study was reviewed and approved by the Human Ethics Committee of the University of Teikyo (trial registration number: 18-233). The medical records of 151 female patients with unilateral endometrioma, including 58 right-sided and 93 left-sided cases, from June 1, 2014, to December 31, 2019, were reviewed retrospectively. We defined the former cases as the right-sided endometrioma group (REG, $n=58)$ and the latter cases as the left-sided endometrioma group ( $L E G, n=93$ ). In this study, we did not include patients with recurrent endometriosis. As shown in Figure 1, of the 302 patients who underwent laparoscopic surgery for new onset endometrioma detected during outpatient examinations, 151 were excluded for the following reasons: 95 patients were excluded because they were diagnosed with bilateral endometrioma, and 56 patients were excluded for other reasons, including no cystic lesion detected during surgery ( $\mathrm{n}=$ $23)$; other main ovarian cystic diseases, such as mature cystic teratoma $(n=8)$; ruptured endometrioma $(n=4)$; and prior abdominal surgery $(n=21)$. Since the last factor (prior abdominal surgery) was excluded after excluding other factors, this number may be an underestimation $(n=21)$. Among the 151 remaining cases, we performed 123 laparoscopic cystectomies, including 9 cases performed simultaneously with laparoscopic myomectomies; 9 cases with laparoscopic hysterectomies; 23 laparoscopic salpingo-oophorectomies, including 3 cases performed simultaneously with laparoscopic hysterectomies; and 5 laparoscopic-assisted cystectomies, including 3 cases performed simultaneously with laparoscopic-assisted myomectomies. The following data were collected: 1) size of the ovarian endometrioma before and during surgery; 2) ASRM score (points) [5];3) patient's age at the time of the operation; 4) presence of adhesions predicted before the operation; 5) history of assisted reproductive technology (ART); 6) presence of complicated adenomyomas, uterine fibroids or other ovarian tumours; and 7) serum carbohydrate antigen 125 (CA125) level (U/ml). Magnetic resonance imaging (MRI) was performed in almost all cases (148/151 cases) to assess the size of the endometrioma. MRI was also used for predicting adhesions, especially in the Douglas pouch. In this study, ART included both in vitro fertilization (IVF) and intracytoplasmic sperm injection (ICSI). In these 151 cases, 28 patients used hormone drugs, including oral contraceptives, gonadotropin-releasing hormone agonists and others. However, conflicting results have been reported in terms of the effect of using hormone drugs prior to surgery on ASRM scores [10], so this factor was not considered in the present study.

\section{Classification of endometrial lesions}

To compare the tendency of endometrial lesion spread between patients with right or left ovarian endometrioma, we divided the endometrial lesion cases based on the location of the endometrioma. Briefly, the so-called ASRM score chart was divided into four parts. First, we calculated the total ASRM 
score for the uterine adnexa on the same side as the ovarian endometrioma and excluded the score for the endometrioma itself, as shown in Figure 2-A. Second, a similar calculation was performed for the uterine adnexa on the opposite side of the ovarian endometrioma (Figure 2-B). The remaining endometrial lesions were divided into peritoneal (Figure 2-C) or Douglas pouch endometrial lesions (Figure 2-C). In summary, we classified the ASRM scores into the following four categories: 1) "same endometrial lesions (SELs)", including Ovary-ENDOMETRIOSIS-Superficial, Ovary-ADHESIONS and TubeADHESIONS on the same side as the main ovarian endometrioma; 2 ) "opposite endometrial lesions (OELS)", including Ovary-ENDOMETRIOSIS-Superficial, Ovary-ADHESIONS and Tube-ADHESIONS on the opposite side of the main ovarian endometrioma; 3 ) "peritoneal endometrial lesions (PELs)", including Peritoneum-ENDOMETRIOSIS; and 4) "Douglas pouch endometrial lesions (DELs)", including POSTERIOR CULDESAC OBLITERATION. For illustration purposes, this classification is shown in Figure 2 for REG. The SEL (Figure 2-A), OEL (Figure 2-B), PEL (Figure 2-C) and DEL (Figure 2-C) scores are the total scores in each cell, as indicated by an italicized letter and thick-bordered box. In the patients with left ovarian endometrioma, the SEL and OEL scores were reversed. In this analysis, the theoretical maximum values of SEL, OEL, PEL and DEL were 36, 36, 10 and 40 points, respectively. We not only compared each average value of SEL, OEL, PEL and DEL between REG and LEG patients but also counted each number of patients with these four lesions in both REG and LEG patients.

\section{Statistical analysis}

The primary outcomes included the difference in the ASRM scores detected during laparoscopic surgery between the patients with right- and left-sided endometriomas. This difference was compared using Student's t-test in Microsoft Excel 2016 (Microsoft, DC, USA). To assess the influence of the following 8 factors on the ASRM score, we performed a multivariate logistic regression analysis: 1) "right-sided endometrioma"; 2) "predicted adhesion", which was defined as the detection of the possible presence of adhesion in a patient by a pelvic examination or MRI; 3) "ART history", which was defined as patients who underwent ART; 4) "adenomyoma", which was defined as the current presence of adenomyoma based on clinical images; 5) "uterine fibroid", which was defined as the current presence of a uterine fibroid based on clinical images; 6) "other ovarian tumour", which was defined as the current presence of an ovarian tumour other than endometrioma based on clinical images; 7) "positive marker", which was defined as serum CA125 levels of $35 \mathrm{U} / \mathrm{ml}$ or higher; and 8) "large endometrioma", which was defined as a tumour size of $50 \mathrm{~mm}$ or larger (described in Table 1). All statistical analyses were performed using JMP version 12 for Windows (SAS Institute, Inc., Tokyo, Japan). The data are presented as the means \pm standard deviations. A p-value less than 0.05 was considered statistically significant.

\section{Results}

\section{Patient characteristics}


The 151 patients with unilateral endometrioma comprised 58 right-sided and 93 left-sided cases (REG and LEG). The average endometrioma size measured before and during surgery, patient age and ASRM score were $56.2 \pm 23.2 \mathrm{~mm}, 58.9 \pm 24.4 \mathrm{~mm}, 36.7 \pm 7.2$ years and $36.2 \pm 18.9$ points, respectively. The representative symptoms were dysmenorrhoea $(n=77)$, infertility $(n=51)$, pelvic pain $(n=38)$, coital pain $(n=7)$ and other symptoms, although the symptoms overlapped in some cases.

By comparing REG and LEG in a univariate analysis, the endometrioma sizes before surgery $(63.7 \pm 23.6$ $\mathrm{mm}$ vs. $57.4 \pm 22.3 \mathrm{~mm}, \mathrm{p}=0.10)$ and during surgery $(64.0 \pm 26.6 \mathrm{~mm}$ vs. $55.7 \pm 22.4 \mathrm{~mm}, \mathrm{p}=0.042)$ and the patients' age ( $36.0 \pm 6.6$ years vs. $37.2 \pm 7.5$ years, $p=0.34)$ did not significantly differ. However, a significantly higher ASRM score was detected for REG (41.5 \pm 22.0 points vs. $33.0 \pm 15.9$ points, $p=$ 0.00677).

\section{Influential factors increasing the ASRM scores}

To detect the significant factors associated with endometriosis severity, we compared 8 factors using multivariate logistic regression models (Table 2). We extracted the following 8 factors collected before surgery: 1) "right-sided endometrioma" ( $n=58) ; 2)$ "predicted adhesion" ( $n=32)$, which was defined as the possible presence of an adhesion detected during a pelvic examination or MRI; 3 ) "ART history" ( $n=$ 14), which was defined as patients who underwent ART; 4) "adenomyoma" ( $n=16)$, which was defined as the current presence of adenomyoma based on clinical images; 5$)$ "uterine fibroid" ( $n=60)$, which was defined as the current presence of uterine fibroids based on clinical images; 6 ) "other ovarian tumour" (n $=13$ ), which was defined as the current presence of an ovarian tumour other than endometrioma based on clinical images; 7$)$ "positive marker" ( $n=85)$, which was defined as serum CA125 levels of $35 \mathrm{U} / \mathrm{ml}$ or higher; and 8) "large endometrioma" ( $(=82)$, which was defined as a tumour size of $50 \mathrm{~mm}$ or larger. Multivariate analysis revealed that "adenomyoma" ( $47.3 \pm 23.0$ points vs. $34.9 \pm 18.0$ points, $p=0.0266)$, "right-sided endometrioma" ( $41.5 \pm 22.0$ points vs. $33.0 \pm 15.9$ points, $p=0.0277$ ) and "uterine fibroid" $(40.4 \pm 21.8$ points vs. $33.5 \pm 16.3$ points, $p=0.0332)$ were significant factors for higher ASRM scores. However, the other 5 factors did not show significant differences. These results supported the hypothesis that the laterality of endometrioma affected the severity of endometriosis independent of the other 2 significant factors. Comparisons of REG and LEG revealed the following: 26/58 patients with REG vs. $34 / 93$ patients with LEG exhibited "uterine fibroid" $(p=0.32)$, and 9/58 patients with REG vs. 7/93 patients with LEG exhibited "adenomyoma" $(p=0.12)$.

\section{Laterality of endometrial lesion spread}

To detect the tendency towards endometrial lesion spread, we compared the total values of SEL, OEL, PEL and DEL between REG and LEG patients. The actual maximal values of SEL, OEL, PEL and DEL were 32, 34,7 and 40 points in the REG patients and 32, 9, 6 and 40 points in the LEG patients, respectively. The 
numbers of SEL, OEL, PEL and DEL cases were $51,23,26$ and 30 , respectively, in the REG patients $(n=58)$ and $80,24,45$ and 35 , respectively, in the LEG patients $(n=93)$. The proportion of patients with OEL or DEL was significantly higher among the REG patients than among the LEG patients. When comparing the scores of these 4 lesions, the OEL ( $3.5 \pm 6.9$ points vs. $1.0 \pm 2.1$ points, $p=0.0013)$ and DEL $(9.5 \pm 15.8$ points vs. $4.2 \pm 10.4$ points, $p=0.014$ ) scores were significantly higher among the REG patients. The SEL (7.9 \pm 7.1 points vs. $7.0 \pm 7.0$ points, $p=0.42)$ and PEL $(1.3 \pm 1.8$ points vs. $1.2 \pm 1.7$ points, $p=0.69)$ did not significantly differ.

\section{Discussion}

One classical proposed pathogenic theory, i.e., the retrograde menstruation hypothesis [11], suggests that the tendency towards frequent left-sided disease is due to the presence of the sigmoid colon [9]. In fact, more cases of left-sided endometrioma were detected at our hospital (93 vs. 58) after excluding 95 cases of bilateral endometrioma. In this study, the possible influence of laterality on the severity of endometriosis was also investigated because the stage of endometriosis is considered closely related to its symptoms, especially infertility $[12,13]$. As an index of severity, we compared the ASRM scores of two groups, i.e., REG patients vs. LEG patients. In this comparison, by excluding patients with bilateral endometrioma, we aimed to determine the main endometriosis lesion in each case. As expected, the ASRM score in the REG patients was significantly higher than that in the LEG patients, and this difference reached approximately 10 points $(41.5 \pm 22.0$ points vs. $33.0 \pm 15.9$ points, $p<0.05)$. Similarly, "uterine fibroid" and "adenomyoma" had a significant influence on the high ASRM scores. The reason is likely because endometriosis, adenomyosis and uterine fibroids can co-occur $[1,2,14,15]$. Based on these results from the multivariate analysis, we ensured that the ratios of patients with "uterine fibroid" and "adenomyoma" were not significantly different before subsequently comparing REG and LEG in more detail.

By classifying the endometrial lesions into 4 patterns (SEL, OEL, PEL and DEL), the detailed characteristics of the endometrial lesions were also compared between REG and LEG. In this analysis, we determined that the more frequent endometrial lesions in the Douglas pouch and the left ovary or tube increased the endometriosis severity in the REG (Table 2). Of these lesion types, DEL was detected in over $50 \%$ of the patients in the REG ( $30 / 58$ patients) and had a larger influence on the ASRM score $(9.5 \pm 15.8$ points; Table 2). Unfortunately, the possibility that the main endometrial lesion in these 30 cases was in the Douglas pouch cannot be ruled out because the pathogenesis of endometriosis is multifactorial and endometriosis does not always arise from the ovary [16]. Additionally, because it is difficult to detect endometrial lesions in the Douglas pouch during a simplified outpatient examination, we mainly focused on "endometrioma". However, the anatomic distribution of endometriotic lesions may also be consistent with the retrograde menstruation theory [11]. In addition to some severe symptoms, such as pelvic pain, these lesions are also associated with more difficult surgery [17]; thus, the differentiation between right or left endometrioma is meaningful. 


\section{Conclusions}

We examined the associations between the laterality of ovarian endometrioma and its severity. Patients with right-sided endometrioma tended to have higher ASRM scores than those with left-sided endometrioma. A detailed analysis of the distribution of endometrial lesions showed the possible involvement of anatomical asymmetries in pelvic organs.

\section{Abbreviations}

\section{ASRM}

American Society for Reproductive Medicine, REG:right-sided endometrioma group, LEG:left-sided endometrioma group, ART:assisted reproductive technology, CA125:carbohydrate antigen 125, MRI:magnetic resonance imaging, IVF:in vitro fertilization, ICSI:intracytoplasmic sperm injection, SELs:same endometrial lesions, OELs:opposite endometrial lesions, PELs:peritoneal endometrial lesions, DELs:Douglas pouch endometrial lesions

\section{Declarations}

\section{Ethics approval and consent to participate}

This retrospective study was approved by the Institutional Review Board of Teikyo University. The study registry number, registry name and date of registration are as follows: 18-233, Clinical outcomes and carcinogenic risk of ovarian endometriomas: a retrospective analysis, 2019/3/20.

\section{Consent for publication}

Written informed consent was obtained from the patients for publication of any images. A copy of the written consent form is available for review by the Editor-in-Chief of this journal.

\section{Availability of data and materials}

The authors agree to make all data of this study freely available.

\section{Competing interests}

The authors declare that they have no competing interests.

\section{Funding}

The authors declare that no funding was received for this study.

\section{Authors' contributions}


WI collected and analysed the data and wrote the manuscript. AT and ON supervised the entire work. MH, AT, AS, RM and HT performed all operations. AF and ON determined the methods of all operations and supervised all medical practice. All authors read and approved the final manuscript.

\section{Acknowledgements}

This research was supported by the Mizonokuchi Hospital of Teikyo University in regard to the provision of medical information. The author(s) disclose the receipt of the financial support of the Japan Society for the Promotion of Conventions for the research, authorship, and/or publication of this article.

\section{Author details}

Department of Obstetrics and Gynaecology, University Hospital Mizonokuchi, Teikyo University School of Medicine, Kanagawa, 5-1-1 Kawasaki, Takatsu-ku, Futago, Kanagawa 213-8507, Japan.

\section{References}

1. Huang JQ, Lathi RB, Lemyre M, Rodriguez HE, Nezhat CH, Nezhat C. Coexistence of endometriosis in women with symptomatic leiomyomas. Fertil Steril. 2010;94(2):720-3.

2.

Khan KN, Kitajima M, Hiraki K, Fujishita A, Sekine I, Ishimaru T, et al. Changes in tissue inflammation, angiogenesis and apoptosis in endometriosis, adenomyosis and uterine myoma after GnRH agonist therapy. Hum Reprod. 2010;25(3):642-53.

3.

Kennedy S, Bergqvist A, Chapron C, D'Hooghe T, Dunselman G, Greb R, et al. ESHRE guideline for the diagnosis and treatment of endometriosis. Hum Reprod. 2005;20(10):2698-704.

4.

Sinaii N, Plumb K, Cotton L, Lambert A, Kennedy S, Zondervan K, et al. Differences in characteristics among 1,000 women with endometriosis based on extent of disease. Fertil Steril. 2008;89(3):538-45. 5.

American Society for Reproductive Medicine. Revised American Society for Reproductive Medicine classification of endometriosis: 1996. Fertil Steril. 1997;67(5):817-21.

6.

Bazot M, Darai E. Diagnosis of deep endometriosis: clinical examination, ultrasonography, magnetic resonance imaging, and other techniques. Fertil Steril. 2017;108(6):886-94.

7. 
Singh SS, Suen MW. Surgery for endometriosis: beyond medical therapies. Fertil Steril. 2017;107(3):54954.

8.

Audebert A, Petousis S, Margioula-Siarkou C, Ravanos K, Prapas N, Prapas Y. Anatomic distribution of endometriosis: A reappraisal based on series of 1101 patients. Eur J Obstet Gynecol Reprod Biol. 2018;230:36-40.

9.

Sznurkowski JJ, Emerich J. Endometriomas are more frequent on the left side. Acta Obstet Gynecol Scand. 2008;87(1):104-6.

10.

Yap C, Furness S, Farquhar C. Pre and post-operative medical therapy for endometriosis surgery (Review). Cochrane Database Syst Rev. 2004(3):CD003678.

11.

Sampson JA. Peritoneal endometriosis due to menstrual dissemination of endometrial tissue into the peritoneal cavity. Am J Obstet Gynecol. 1927;14:442-69.

12.

Prefumo F, Rossi AC. Endometriosis, endometrioma, and ART results: Current understanding and recommended practices. Best Pract Res Clin Obstet Gynaecol. 2018;51:34-40.

13.

Isono W, Wada-Hiraike O, Shirane A, Fujimoto A, Osuga Y, Yano T, et al. Alternative strategies to in vitro fertilization/intracytoplasmic sperm injection treatment for aged infertile women. Reprod Med Biol. 2012;11(1):69-72.

14.

Naphatthalung W, Cheewadhanaraks S. Prevalence of endometriosis among patients with adenomyosis and/or myoma uteri scheduled for a hysterectomy. J Med Assoc Thai. 2012;95(9):1136-40. 15.

Isono W, Wada-Hiraike O, Osuga Y, Yano T, Taketani Y. Diameter of dominant leiomyoma is a possible determinant to predict coexistent endometriosis. Eur J Obstet Gynecol Reprod Biol. 2012;162(1):87-90. 16.

Osuga Y. Current concepts of the pathogenesis of endometriosis. Reprod Med Biol. 2010;9(1):1-7. 17.

Arion K, Aksoy T, Allaire C, Noga H, Williams C, Bedaiwy MA, et al. Prediction of Pouch of Douglas Obliteration: Point-of-care Ultrasound Versus Pelvic Examination. J Minim Invasive Gynecol. 2018.

\section{Tables}

Table 1: Influence on the ASRM score. 


\begin{tabular}{llcc}
\hline Factors & $\mathrm{N}$ & ASRM score & P-value \\
\hline Adenomyoma & 16 & $47.3 \pm 23.0$ vs. $34.9 \pm 18.0$ & 0.0266 \\
\hline Right-side Endometrioma & 58 & $41.5 \pm 22.0$ vs. $33.0 \pm 15.9$ & 0.0277 \\
\hline Uterine fibroid & 60 & $40.4 \pm 21.8$ vs. $33.5 \pm 16.3$ & 0.0332 \\
\hline Large endometrioma & 82 & $37.3 \pm 18.7$ vs. $35.0 \pm 19.2$ & 0.5019 \\
\hline ART history & 14 & $40.0 \pm 14.8$ vs. $35.9 \pm 19.3$ & 0.5458 \\
\hline Other ovarian tumour & 13 & $36.3 \pm 20.9$ vs. $36.2 \pm 18.8$ & 0.7469 \\
\hline Predicted adhesion & 32 & $36.0 \pm 14.9$ vs. $36.3 \pm 19.9$ & 0.7980 \\
\hline Positive marker & 85 & $37.0 \pm 18.0$ vs. $35.2 \pm 20.1$ & 0.8532 \\
\hline
\end{tabular}

Footnote

ART: assisted reproductive technology

Table 2: Distribution of endometrial lesions.

\begin{tabular}{lccc}
\hline Lesion & Right side & Left side & $\mathrm{P}$ \\
\hline OEL & $3.4 \pm 6.9(\mathrm{n}=23)$ & $1.0 \pm 2.1(\mathrm{n}=24)$ & 0.0013 \\
\hline DEL & $9.5 \pm 15.8(\mathrm{n}=30)$ & $4.2 \pm 10.4(\mathrm{n}=35)$ & 0.014 \\
\hline SEL & $7.9 \pm 7.1(\mathrm{n}=51)$ & $7.0 \pm 7.0(\mathrm{n}=80)$ & 0.42 \\
\hline PEL & $1.3 \pm 1.8(\mathrm{n}=26)$ & $1.2 \pm 1.7(\mathrm{n}=45)$ & 0.69 \\
\hline
\end{tabular}

Footnote

OEL: Opposite endometrial lesion, DEL: Douglas pouch endometrial lesion, SEL: Same endometrial lesion, PEL: Peritoneal endometrial lesion

\section{Figures}


Figure 1: Flowchart of data collection criteria.

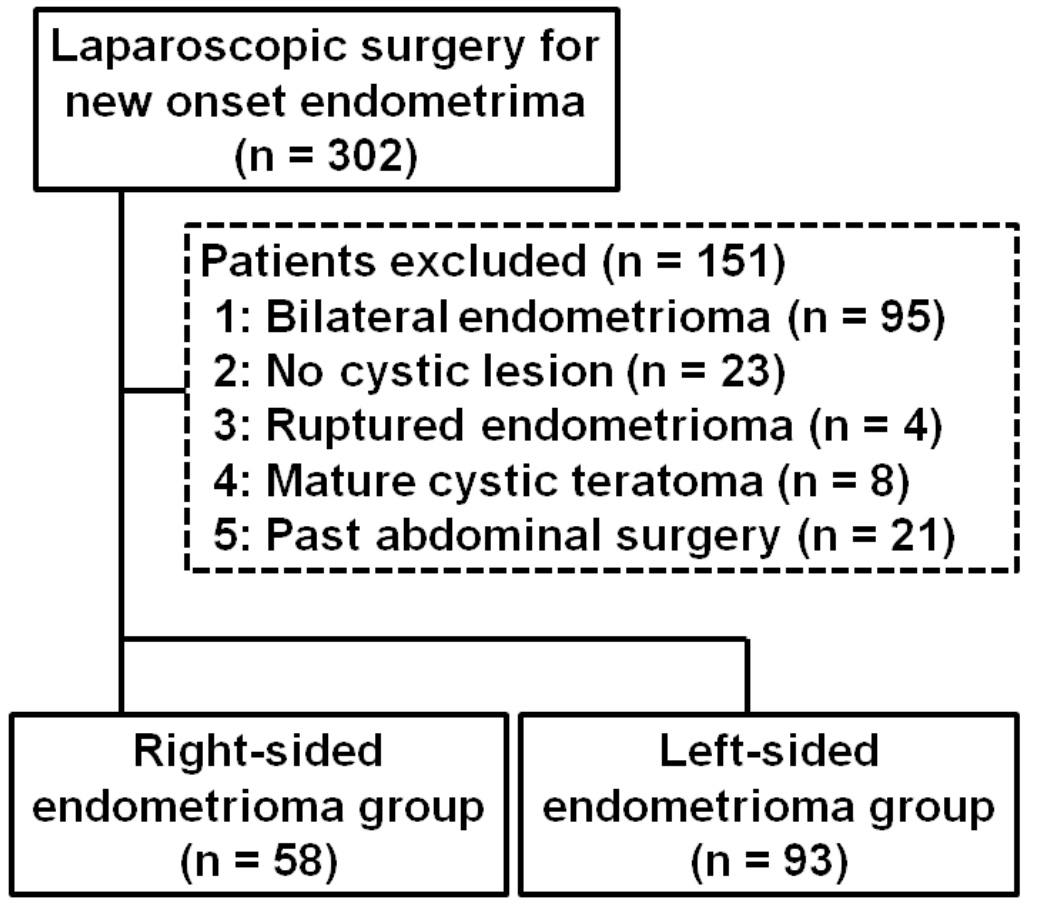

Figure 1

Flowchart of data collection criteria. Of the 302 patients, 151 were excluded for the following reasons: 95 patients were excluded because they were diagnosed with bilateral endometrioma, and 56 patients were excluded for other reasons, including no cystic lesion detected during surgery $(n=23)$; other main ovarian cystic diseases, such as mature cystic teratoma $(n=8)$; ruptured endometrioma $(n=4)$; and past abdominal surgery $(n=21)$. This last factor (past abdominal surgery) was excluded after excluding other 
factors. Then, the medical records of 151 female patients with unilateral endometrioma, including 58 right-sided and 93 left-sided cases, were reviewed retrospectively.

Figure 2: Classification of endometrial lesions in patients with right-side endometrioma
A. Same endometrial lesion (SEL)
B. Opposite endometrial lesion (OEL)

\begin{tabular}{|c|c|c|c|c|}
\hline \multirow{3}{*}{ 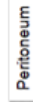 } & ENDOMETRIOSIS & $<1 \mathrm{~cm}$ & $1.3 \mathrm{~cm}$ & $>3 \mathrm{~cm}$ \\
\hline & Superficial & 1 & 2 & 4 \\
\hline & Deep & 2 & 4 & 6 \\
\hline \multirow{4}{*}{ 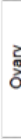 } & R Superficial & 1 & 2 & 4 \\
\hline & Deep & 4 & 16 & 20 \\
\hline & L Superficial & 1 & 2 & 4 \\
\hline & Deep & 4 & 16 & 20 \\
\hline \multirow{2}{*}{\multicolumn{2}{|c|}{$\begin{array}{l}\text { POSTERIOR CULDESAC } \\
\text { OBLITERATION }\end{array}$}} & \multicolumn{2}{|l|}{ Partial } & Complete \\
\hline & & \multicolumn{2}{|l|}{4} & 40 \\
\hline \multirow{5}{*}{ రై } & ADHESIONS & $<1 / 3$ Enclosure & 1/3-2/3 Enclosure & $>2 / 3$ Enclosure \\
\hline & R Filmy & 1 & 2 & 4 \\
\hline & Dense & 4 & 8 & 16 \\
\hline & L Filmy & 1 & 2 & 4 \\
\hline & Dense & 4 & 8 & 16 \\
\hline \multirow{4}{*}{ 胥 } & R Filmy & 1 & 2 & 4 \\
\hline & Dense & 4 & 8 & 16 \\
\hline & L Filmy & 1 & 2 & 4 \\
\hline & Dense & 4 & 8 & 16 \\
\hline
\end{tabular}

\begin{tabular}{|c|c|c|c|c|}
\hline \multirow{3}{*}{ 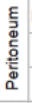 } & ENDOMETRIOSIS & $<1 \mathrm{~cm}$ & $1-3 \mathrm{~cm}$ & $>3 \mathrm{~cm}$ \\
\hline & Superficial & 1 & 2 & 4 \\
\hline & Deep & 2 & 4 & 6 \\
\hline \multirow{4}{*}{ ठे } & R Superficial & 1 & 2 & 4 \\
\hline & Deep & 4 & 16 & 20 \\
\hline & L Superficial & 1 & 2 & 4 \\
\hline & Deep & 4 & 16 & 20 \\
\hline \multirow{2}{*}{\multicolumn{2}{|c|}{$\begin{array}{l}\text { POSTERIOR CULDESAC } \\
\text { OBLITERATION }\end{array}$}} & \multicolumn{2}{|l|}{ Partial } & Complete \\
\hline & & \multicolumn{2}{|l|}{4} & 40 \\
\hline \multirow{5}{*}{$\begin{array}{l}\text { टे } \\
\text { ổ }\end{array}$} & ADHESIONS & $<1 / 3$ Enclosure & 1/3-2/3 Enclosure & $>2 / 3$ Enclosure \\
\hline & R Filmy & 1 & 2 & 4 \\
\hline & Dense & 4 & 8 & 16 \\
\hline & L Filmy & 1 & 2 & 4 \\
\hline & Dense & 4 & 8 & 16 \\
\hline \multirow{4}{*}{ 今̊ } & R Filmy & 1 & 2 & 4 \\
\hline & Dense & 4 & 8 & 16 \\
\hline & L Filmy & 1 & 2 & 4 \\
\hline & Dense & 4 & 8 & 16 \\
\hline
\end{tabular}

\begin{tabular}{|c|c|c|c|c|}
\hline \multirow{3}{*}{ 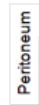 } & ENDOMETRIOSIS & $<1 \mathrm{~cm}$ & $1.3 \mathrm{~cm}$ & $>3 \mathrm{~cm}$ \\
\hline & Superficial & 1 & 2 & 4 \\
\hline & Deep & 2 & 4 & 6 \\
\hline \multirow{6}{*}{ वृ } & $R$ Superficial & 1 & 2 & 4 \\
\hline & Deep & 4 & 16 & 20 \\
\hline & L Superficial & 1 & 2 & 4 \\
\hline & Deep & 4 & 16 & 20 \\
\hline & \multirow{2}{*}{$\begin{array}{l}\text { POSTERIOR CULDESAC } \\
\text { OBLITERATION }\end{array}$} & \multicolumn{2}{|l|}{ Partial } & omplete \\
\hline & & \multicolumn{2}{|l|}{4} & 40 \\
\hline \multirow{5}{*}{ ठิ } & ADHESIONS & $<1 / 3$ Enclosure & 1/3-2/3 Enclosure & $>2 / 3$ Enclosure \\
\hline & R Filmy & 1 & 2 & 4 \\
\hline & Dense & 4 & 8 & 16 \\
\hline & L Filmy & 1 & 2 & 4 \\
\hline & Dense & 4 & 8 & 16 \\
\hline \multirow{4}{*}{ 总 } & R Filmy & 1 & 2 & 4 \\
\hline & Dense & 4 & 8 & 16 \\
\hline & L Filmy & 1 & 2 & 4 \\
\hline & Dense & 4 & 8 & 16 \\
\hline
\end{tabular}

C. Peritoneal endometrial lesion (PEL) and Douglas pouch endometrial lesion (DEL)

\section{Figure 2}

Classification of endometrial lesions in patients with right-sided endometrioma. In addition to the right or left endometriomas treated generally as deep endometriosis, we classified the ASRM score into the following four categories: 1) "Same endometrial lesions (SELs)", including ovarian and tubal endometrial 
lesions on the same side as the main ovarian endometrioma; 2) "Opposite endometrial lesions (OELs)", including ovarian and tubal endometrial lesions on the opposite side of the main ovarian endometrioma; 3) "Peritoneal endometrial lesions (PELs)"; and 4) "Douglas pouch endometrial lesions (DELs)". For illustration, the classification of SEL (A), OEL (B), PEL (C) and DEL (C) in the REG is shown using the Chart of Revised ASRM Score [5]. The scores of SEL, OEL, PEL and DEL are the total values of the scores in each cell as indicated by an italic letter and thick-bordered box. In the LEG, the scores of SEL and OEL are reversed. 